\title{
Retrospective Analysis of Chinese Patients with Hepatocellular Carcinoma (HCC) undergoing Transcatheter Arterial Chemoembolization (TACE) with or without Prophylactic Antibiotic Therapy
}

\author{
Jiu Chen and Guolin Wu
}

\begin{abstract}
Objective: To assess the necessity of prophylactic antibiotic therapy in HCC patients undergoing transcatheter arterial chemoembolization (TACE).

Study Design: Descriptive study.

Place and Duration of Study: The First Affliated Hospital, College of Medicine, Zhejiang University, Hangzhou, China, from August 2016 to August 2017.

Methodology: Retrospective TACE-treated cases were analysed in this study. The basic characteristics of the patients, the days in hospital, the laboratory data and fever after the procedure were assessed and compared. A review of literature of published studies and our present study were further conducted to determine the necessity of prophylactic antibiotic therapy.

Results: In 159 cases, there were no significant difference in the leukocyte count $(p=0.552)$, neutrophil count $(p=0.573)$ and fever $(p=0.31)$ with and without prophylactic antibiotic therapy groups. The hospital stay was significantly increased in the prophylactic antibiotic group $(p<0.001)$. The fever of previous and the present results showed no significant difference $(p=0.25)$ between the groups.

Conclusion: Prophylactic antibiotic therapy is not routinely necessary in the procedure of TACE for HCC patients.
\end{abstract}

Key Words: Transcatheter arterial chemoembolization, Hepatocellular carcinoma, Antibiotic prophylaxis.

\section{INTRODUCTION}

Hepatocellular carcinoma $(\mathrm{HCC})$ is one of the most common maligancies all over the world, ${ }^{1}$ especially in the areas with the background of large amount of HBV and cirrhosis diseases, such as China. Transcatheter arterial chemoembolization (TACE), with its effectiveness and safety, is now the most widely used treatment of $\mathrm{HCC}$ in the patients among the various strategies. ${ }^{2}$ Like other invasive procedures, TACE also takes the risk of bacterial infection, especially in the patients of chronic HBV infection and cirrhosis, and the potentially fatal infection like abscess have been reported. ${ }^{3}$ But, it is still controversial in the use of prophylaxis antibiotic in the procedure. Reviews in 2017 pointed that the antibiotics is necessary and beneficial even in the absence of risk of inflectional factors. ${ }^{4}$ Other analysis of previous clinical trials, 5 pointed that the use of antibiotics may not be routinely necessary.

\footnotetext{
Department of Traditional Chinese Medicine, The First Affliated Hospital, College of Medicine, Zhejiang University, Hangzhou, China

Correspondence: Dr. Guolin Wu, Department of Traditional Chinese Medicine, The First Affliated Hospital, College of

Medicine, Zhejiang University, Hangzhou, China

E-mail:linyunju@zju.edu.cn

Received: March 09, 2018; Accepted: July 20, 2018
}

The abuse of antibiotics is now a worldwide problem, it not only increases the expenditures and the probability of the adverse drug reactions (ADR), but also was thought one of the main reason of the emergence of more and more antibiotic-resistant strains of organisms. It is necessary and urgent to optimise the antibiotic treatment strategy in the procedure of TACE, not only for the HCC patients but also for the entire population.

By analyzing the available clinical trials and the present retrospectively analysis of 159 cases in the past year, the aim of this study was to assess the necessity of prophylactic antibiotic therapy in HCC patients undergoing TACE.

\section{METHODOLOGY}

HCC cases undergoing TACE at the First Affiliated Hospital, College of Medicine, Zhejiang University from August 2016 to August 2017 were enrolled in this retrospective analysis. This study was approved by the Ethical Committee of the Institute.

The inclusion criteria were patients with the evidence of hepatitis B virus infection, cirrhosis and HCC. The exclusion criteria were patients with severe cardiovascular, hematologic, renal disease or other important organ diseases including the metastatic lung tumors from the liver, metastatic liver tumors, neuroendocrine 
tumors and other histological malignancies, cholangioenterostomy (Roux-en-Y), biliary stent implantation or other kind of biliary abnormalities, leukocyte count lower than $2 \times 109 / \mathrm{L}$ or higher than $10 \times 109 / \mathrm{L}$ count, the neutrophil lower than $1 \times 109 / \mathrm{L}$ before TACE, and the level of serum albumin lower than $30 \mathrm{~g} / \mathrm{L}$.

Cholangioenterostomy (Roux-en-Y), biliary stent implantation or other kind of biliary abnormalities; leukocyte count lower than $2 \times 10^{9} / \mathrm{L}$ or higher than $10 \times 10^{9} / \mathrm{L}$ count, the neutrophil lower than $1 \times 10^{9} / \mathrm{L}$ before TACE, and the level of serum albumin lower than $30 \mathrm{~g} / \mathrm{L}$. Patients were also excluded if they were on any medicines before TACE which could affect the tolerance of infection, clinical manifestations or serum indices after TACE; if other procedures were taken in the same duration of the hospital day like partial splenic embolization, trans-jugular intrahepatic portosystemic stent shunt, resection, radio frequency ablation, etc.; or when infections were existed before TACE, such as lung infection, urinary infection and so on.

Based on the antibiotics used or not, patients were assigned to the prophylactic antibiotic group and the blank group. In the prophylactic antibiotic group, prophylactic antibiotic therapy was administered on the day of TACE and sustained for at least 48 hours. In the prophylactic antibiotic group, different antibiotics were used alone or combined. In the blank group, no prophylactic antibiotic therapy was administered after TACE. The antibiotic administration period could be prolonged, or its dose could be altered according to the physician's judgment.

Patients were assessed before treatment. The leukocyte and neutrophil counts were collected on the next morning after TACE. The leukocyte and neutrophil counts, the days in hospital and fever $\left(>38^{\circ} \mathrm{C}\right)$ before discharge after the treatment were gathered and evaluated to compare the effectiveness of the antibiotic prophylaxis.

Every patient referred for TACE treatment was first evaluated by an interventional radiologist. After fasting for at least four hours, the patient was brought to the special suite. To better delineate the blood supply of the tumor, diagnostic visceral and selective hepatic arteriography were performed after obtaining the femoral artery. After identifying the hypervascular tumor blush, the feeding vessels of the tumor were selectively catheterized. The therpentic agents including antiemetic, chemotherapeutic agents, ethiodol and/or hepasphereloaded micropheres, which were calculated before procedure according to the state of the patients, were administered to reduce the arterial inflow of the tumor. The procedure was deemed technically successful when the entire amount of chemotherapeutic agent was delivered to the tumor or when there was stagnation of flow or evidence of reflux into the main artery. Technical success of the chemoembolization procedure was confirmed by the presence of intense tumor staining images obtained immediately after the procedure.

Mean \pm SD for continuous variables and frequency with percentages for qualitative variables; differences in the distribution of baseline characteristics as well as infection indices between cases and controls were assessed using Student's t-test for continuous variables and Pearson $\chi 2$-test for categorical variables. The comparisons before and after treatment were evaluated using the paired Student's t-test. All statistical analyses were performed using the SPSS software (version 23.0). The $p$-value $<0.05$ was considered statistically significantly.

Following a preliminary literature survey, an electronic search was performed using the MeSH terms "carcinoma, hepatocellular", "chemoembolization, therapeutic" and "antibiotic prophylaxis" or "infection", which included searches in the PubMed, Web of Science, Cochrane Library, Chinese Hospital Knowledge Databases (CHKD) and Wang Fang Med Online. Only clinical trials and full texts available were considered for inclusion. In addition, the reference lists of the retrieved articles were manually screened for other potentially relevant studies. An analysis of these four studies (three studies, $6-8$ and the present study) as conducted, which included 672 cases and 277 controls to further assess the association between fever and antibiotic therapy in HCC patients undergoing TACE and Begg's funnel plot was evaluated.

\section{RESULTS}

There were no significant differences between the two groups in terms of baseline characteristics before TACE (Table I).

The count of serum leukocyte and neutrophil before and after TACE is presented in Table II. TACE increased leukocyte and neutrophil the counts in both groups. The indices after TACE are presented in Table III. There were

Table I: Baseline characteristics of the patients.

\begin{tabular}{l|c|c|c}
\hline Variables & $\mathrm{AB}$ & $\mathrm{NO} A \mathrm{~B}$ & $\mathrm{p}$-value \\
\hline Number of patients & $87(54.7 \%)$ & $72(45.3 \%)$ & \\
\hline Age (years) & $57.8 \pm 10.39$ & $60.18 \pm 10.57$ & 0.156 \\
\hline Medium range & & & \\
\hline Sex & $80(92 \%)$ & $61(84.7 \%)$ & 0.152 \\
Male & $7(8 \%)$ & $11(15.3 \%)$ & \\
Female & $78(89.7 \%)$ & $67(93.1 \%)$ & 0.451 \\
\hline Diabetes mellitus & $9(10.3 \%)$ & $5(6.9 \%)$ & \\
No & & & \\
Yes & $37.86 \pm 3.922$ & $38.63 \pm 4.158$ & 0.229 \\
\hline Serum index before procedure & $14.94 \pm 6.956$ & $13.61 \pm 5.693$ & 0.195 \\
Albumin & $12.313 \pm 0.985$ & $12.293 \pm 0.966$ & 0.900 \\
Total bilirubin & $4.594 \pm 1.461$ & $4.460 \pm 1.554$ & 0.575 \\
Prothrombintime & $2.901 \pm 1.170$ & $2.768 \pm 1.117$ & 0.467 \\
Leukocyte & & & \\
Neutrophil & AB & &
\end{tabular}


no significant differences in the fever, the count of serum leukocyte and neutrophil between the two groups, but the days in hospital were obviously increased in the prophylactic antibiotics group.

The evaluated studies were conducted between January 2001 and August 2017. Begg's funnel plot did not reveal remarkable asymmetry in the distribution of scatter points $(p=0.0641$, Figure 1$)$. Heterogeneity among four studies was not statistically significant $(p=0.25,12=27 \%)$.

Table II: Comparison of leukocyte and neutrophil count before and after

\begin{tabular}{l|c|c|c}
\multicolumn{2}{c}{ TACE. } \\
\hline Variables & Before TACE & After TACE & p-value \\
\hline Leukocyte count & & & \\
AB & $4.594 \pm 1.461$ & $6.976 \pm 2.887$ & $<0.001$ \\
NO AB & $4.460 \pm 1.554$ & $6.708 \pm 2.737$ & $<0.001$ \\
\hline Neutrophil count & & & \\
AB & $2.901 \pm 1.170$ & $5.740 \pm 2.669$ & $<0.001$ \\
NO AB & $2.768 \pm 1.117$ & $5.504 \pm 2.563$ & $<0.001$ \\
\hline
\end{tabular}

$A B=$ Antibiotic therapy; $\quad N O A B=$ No antibiotic therapy

Table III: The indices after TACE.

\begin{tabular}{l|c|c|c}
\hline Variables & $\mathrm{AB}$ & $\mathrm{NO} A \mathrm{~B}$ & $\mathrm{p}$-value \\
\hline No fever $\left(\leq 38.0^{\circ} \mathrm{C}\right)$ & $71(81.6 \%)$ & $63(87.5 \%)$ & 0.310 \\
Fever $\left(>38.0^{\circ} \mathrm{C}\right)$ & $16(18.4 \%)$ & $9(12.5 \%)$ & \\
Leukocyte count & $6.976 \pm 2.887$ & $6.708 \pm 2.737$ & 0.552 \\
Neutrophil count & $5.740 \pm 2.670$ & $5.504 \pm 2.563$ & 0.573 \\
Days in hospital & $6.44 \pm 2.084$ & $5.06 \pm 1.775$ & $<0.001$ \\
\hline
\end{tabular}

$A B=$ Antibiotic therapy; $\quad N O A B=$ No antibiotic therapy

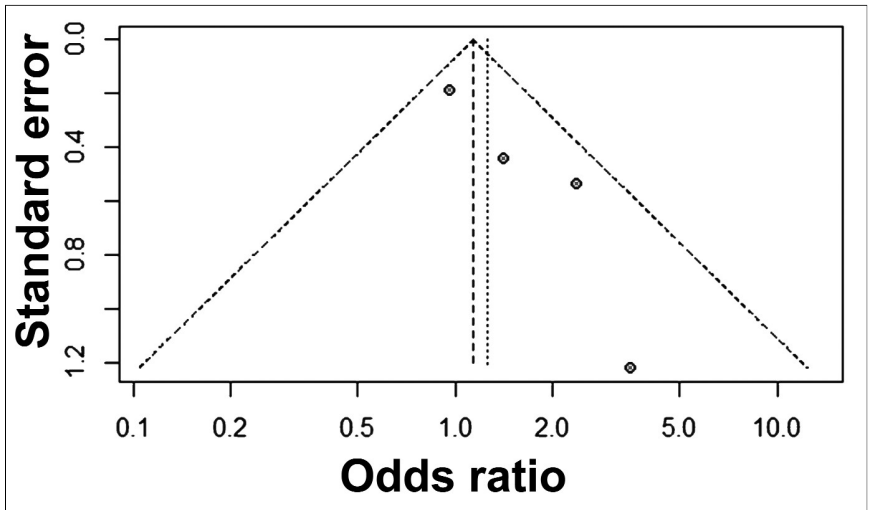

Figure 1: Begg's funnel plot of analysis.
No significant association $(p=0.25)$ between fever and antibiotic therapy in HCC patients undergoing TACE was observed in either comparison under the fixed effect model (OR=1.15, $95 \% \mathrm{Cl}: 0.84-1.58)$ or the random effects model ( $\mathrm{OR}=1.32,95 \% \mathrm{Cl}$ : 0.81-2.16).

The four studies assessing the effect of prophylactic antibiotic treatment in HCC patients were showed in Figure 2. By analysis of frequency of fever in previous and the present study, there was still no significant $(p=0.25)$ difference between the groups.

\section{DISCUSSION}

Liver cancer is the fifth most common cancer and the second most common cause of cancer-related death worldwide, and the burden in China alone accounts for approximately $50 \%$ of the total number of cases and deaths. ${ }^{1} \mathrm{HCC}$ accounts for up to $90 \%$ of all liver cancer cases. ${ }^{1}$ The pathogenesis of HCC is still unknown. It always develops in the background of the chronic liver diseases. With 0.95 ratio of mortality to incidence, ${ }^{1} \mathrm{HCC}$ has very poor prognosis and is fatal if not treated. Liver transplant, partial hepatectomy, and local regional ablation were thought the potentially curative therapies in the treatment of $\mathrm{HCC}$. With the insidious onset and various clinical appearances, less than $20 \%$ of HCC patients are found to be curative at presentation. ${ }^{9}$ The patients received the curative therapies, and the longterm prognosis after surgical resection of $\mathrm{HCC}$ remains unsatisfactory for the high incidence of recurrence and metastasis. 10

TACE is a 2-step procedure that involves selection injection of 1 or more chemotherapeutic agents and insertion of embolic material into the feeding arteries of the tumor. It has been used since the 1980s and is now the mainstay of therapy in patients with lesions which are not suitable for these above potentially curable treatments, prior to surgery or transplantation and after the resection of HCC (adjuvant TACE). 11 TACE has demonstrated its excellent effectiveness in improving in quality of life, overall survival time and adequate preservation of liver function. ${ }^{2}$

Although it is usually well tolerated, TACE also carries the fatal risk of bacterial infection as a kind of invasive

\begin{tabular}{|c|c|c|c|c|c|c|c|c|c|c|c|}
\hline Study & $\begin{array}{l}\text { Experim } \\
\text { Events }\end{array}$ & $\begin{array}{l}\text { ental } \\
\text { Total }\end{array}$ & & $\begin{array}{l}\text { ntrol } \\
\text { Total }\end{array}$ & & Odds Ratio & & OR & $95 \%-\mathrm{Cl}$ & $\begin{array}{r}\text { Weight } \\
\text { (fixed) }\end{array}$ & $\begin{array}{r}\text { Weight } \\
\text { (random) }\end{array}$ \\
\hline Plentz 2005 & 3 & 15 & 1 & 15 & & $\because$ & & 3.50 & [0.32; 38.23] & $1.1 \%$ & $4.0 \%$ \\
\hline Gao 2013 & 15 & 56 & 6 & 45 & & t: & & 2.38 & {$[0.84 ; 6.75]$} & $6.8 \%$ & $17.4 \%$ \\
\hline Zhu 2014 & 239 & 514 & 69 & 145 & & 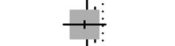 & & 0.96 & {$[0.66 ; 1.38]$} & $80.8 \%$ & $56.2 \%$ \\
\hline Chen 2017 & 16 & 87 & 9 & 72 & & 7 & & 1.58 & {$[0.65 ; 3.82]$} & $11.3 \%$ & $22.4 \%$ \\
\hline \multirow{3}{*}{$\begin{array}{l}\text { Fixed effect model } \\
\text { Random effects model } \\
\text { Heterogeneity: } I^{2}=27 \%, \tau^{2}\end{array}$} & & & & 277 & & & & $\begin{array}{l}1.15 \\
1.32\end{array}$ & $\begin{array}{l}{[0.84 ; 1.58]} \\
{[0.81 ; 2.16]}\end{array}$ & $\begin{array}{r}100.0 \% \\
--\end{array}$ & $100 . \overline{-}$ \\
\hline & $\tau^{2}=0.0757$, & $p=0$ & & & $\Gamma$ & 1 & 7 & & & & \\
\hline & & & & & 0.1 & 0.512 & 10 & & & & \\
\hline
\end{tabular}

Figure 2: Forest plot of 4 studies assessing the effect of prophylactic antibiotic treatment in HCC patients. 
procedure and the use of antibiotics at the beginning of application was thought not only to decrease the sepsis but also allow for the use of high concentrations of chemotherapeutic agents. ${ }^{12}$ The infection can be caused by the bacteria from the skin to the blood via the puncture, the bacteria reflux via the biliary system from the intestine to the liver and so on. Although the severe incidence of infection like liver abscess after TACE was $0.58 \%$ per patient and had $0.19 \%$ per procedure, 5 once the post-procedure infectious complications occurred, the condition of some patients can be devastating. ${ }^{13}$ And the serious infectious complications like sepsis and liver abscess can happen sometimes. In three separate studies with large samples, three abscesses were seen in a series of 1,348 cases, 14 Seven abscesses each were seen in a series of 827 TACE sessions, 15 and a series of 2,581 cases. ${ }^{16}$ Bacterial infection seems more likely to occur in patients with prior bilioenteric anastomosis, previous sphincterotomy, biliary drainage, biliary stent implantation and diabetes, 3,16 and the previous biliary abnormalities were the most mentioned risks the incidence of liver abscess was up to $57.1 \%, 3$ and the mortality rate of abscess could be up to $50 \% .{ }^{17}$

Leukopenia, which is common in the patients with cirrhosis, is another risk factor of bacterial infection. It seems that prophylactic antibiotic treatment is necessary as the fatal infection may exist in $\mathrm{HCC}$ patients with the background of HBV and cirrhosis which means the decreased resistance to bacterial infection because of the hepatic functional damage, leukopenia and so on. ${ }^{18}$ Reviews in 2017 pointed that the antibiotics is necessary and beneficial even in the absence of risks described above. ${ }^{4}$ The primary mechanism for infection was thought most likely contamination of the embolized segment of hepatic parenchyma with biliary pathogens. ${ }^{13}$ But the analysis of previous clinical trials pointed that the use of antibiotics may not be routinely necessary. 5

Although diabetes mellitus is considered a risk factor for bacterial infections, neither the nine diabetics in the antibiotic group nor the five diabetics in the blank group had bacterial infection during hospital stay.

Antibiotics may be abused or improperly selected, increasing not only the medical expenditures and probability of ADR, but also the bacterial resistance of the entire population. The use of antibiotics is an important factor contributing to the emergence of antibiotic resistance. As the mobility of the entire population is continuously increasing, once the antibiotic-resistant strains of organisms emerged, the spread seems more likely than before. Therefore, the use and abuse of antibiotics is a growing public health concern worldwide as the emergence of more and more antibiotic-resistant strains of organisms; especially the multidrug-resistant organisms, which may be life-threatening and are difficult to manage. According to the USA's National Nosocomial Infections Surveillance System, the rate of methicillin-resistant MRSA has been nearly doubled over the last decade in the intensive care units and the gram-negative organisms such as Acinetobacter baumannii, Pseudomonas aeruginosa and extended-spectrum beta lactamase-producing enterobacteriaceae demonstrate increasing resistance to the third-generation cephalosporins, fluoroquinolones, and even carbapenems in some cases. As the incidence of infections caused by antimicrobial-resistant organisms continue to increase and adverse consequences are serious, we should use the antibiotics with extreme caution in every procedure; otherwise, clinician may be faced with a growing number of potentially fatal infections in the not-too-distant future.

Fever and elevated leukocyte are the common manifestations of bacterial infection in clinical, but other reasons can also induce both fever and elevated leukocyte. After the course of TACE, fever and elevated leukocyte are the most common manifestations in clinical. A meta-analysis pointed that the incidence of fever after TACE is up to $80 \%, 19$ while severe incidence of infection like liver abscess was only $0.58 \%$ per patient and $0.19 \%$ per procedure, as described above. In most conditions, fever was not the manifestation of bacterial infection but thought to be caused mainly by the tissue ischemia induced by the chemoembolization and the consequent cytokine release and inflammatory response.20 Neutrophil is only one kind of leukocyte. Only a small amount of leukocytes are in the blood circulation of the body; the others can move to the blood as needed. Except for the bacterial infections, the level of leukocytes in the blood can be increased in both physiological and other pathophysiological conditions, such as exercise, pregnancy, sterile inflammatory response and so on. The inflammatory response was induced by the tissue damage and necrosis during the course of TACE. This may be the main reason of the increased level of neutrophil and leukocytes in the study.

The decision of prophylactic antibiotic therapy may be subjective and empirical. In cases, when fever or elevated leukocyte exist, the physicians may use antibiotics empirically, as per individualised choice of antibiotics.

The result of this study may have some bias because of the retrospective way and limited samples, so more samples and more indices of bacterial infection in prospective studies are needed to verify the result.

\section{CONCLUSION}

The analysis of the present study suggested that the prophylactic antibiotic therapy is not routinely necessary in the procedure of TACE for HCC patients. 


\section{REFERENCES}

1. Ferlay J, Soerjomataramll, Dikshit R. Cancer incidence and mortality worldwide: Sources, methods and major patterns in GLOBOCAN 2012. Int J Cancer 2015; 136:E359-86.

2. Llovet JM, Real MI, Montaña X. Arterial embolisation or chemoembolisation versus symptomatic treatment in patients with unresectable hepatocellular carcinoma: A randomised controlled trial. Lancet 2002; 359:1734-9.

3. Lv F, Lu D, He YS, Xiao JK, Zhou CZ, Cheng DL. Liver abscess formation following transarterial chemoembolization: Clinical features, risk factors, bacteria spectrum, and percutaneous catheter drainage. Medicine 2016; 95:e3503.

4. Taslakian B, Sridhar D. Post-procedural care in interventional radiology: What every interventional radiologist should knowPart I: Standard post-procedural instructions and follow-up care. Cardiovasc Intervent Radiol 2017; 40:481-95.

5. Li YJ, Li GW, Huang XF, Zeng QA. Clinical significance of antibiotic prophylaxis in transarterial therapy of hepatocellular carcinoma: A meta- analysis. Chin Arch Gen Surg (Electronic Edition) 2016; 10:231-4.

6. Plentz RR, Lankisch TO, Bastürk M. Prospective analysis of German patients with hepatocellular carcinoma undergoing transcatheter arterial chemoembolization with or without prophylactic antibiotic therapy. J Gastroenterol Hepatol 2005; 20:1134-6.

7. Gao F, Zhang XN, Chen MZ, Qian T, Ma AY, Yin HB. Prophylactic use of antibiotics after interventional procedures for primary hepatocellular carcinoma: Its clinical significance. J Intervent Radiol 2013; 22:151-3.

8. Zhu WY, Liu JY, Zhou YH, Liu L, Feng S, Gao B. Discussion of prophylactic use of antibiotics in interventional procedures for primary hepatocellular carcinoma. Shandong Medi J 2014; 54: 59-61.

9. Shao JY, LeeF, Chang CL, Wu SY. Statin-based palliative therapy for hepatocellular carcinoma. Medicine (Baltimore) 2015; 94:e1801.

10. Li F, Guo Z, Wang H. Influencing elements and treatment strategies associated with the relapse of hepatocellular carcinoma after surgery. Hepatogastroenterology 2013; 60: 1148-55

11. Li KW, Li X, Wen TF, Lu WS. The effect of postoperative TACE on prognosis of HCC: An update. Hepatogastroenterology 2013; 60:248-51.

12. Reed RA, Teitelbaum GP, Daniels JR, PentecostMJ, Katz MD. Prevalence of infection following hepatic chemoembolization with cross-linked collagen with administration of prophylactic antibiotics. J Vasc Interv Radiol 1994; 5:367-71.

13. Darragh FH, William CT. The infectious complications of interventional radiology based procedures in gastroenterology and hepatology. J Gastrointestin Liver Dis 2011; 20:71-5.

14. Xia JL, Ren ZG, Ye SL. Study of severe and rare complications of transarterial chemoembolization (TACE) for liver cancer. Eur J Radiol 2006; 59:407-12.

15. Tarazov PG, Polysalov VN, Prozorovskij KV, Grishchenkova IV, Rozengauz EV. Ischemic complications of transcatheter arterial chemoembolization in liver malignancies. Acta Radiol 2000; 41:156-60.

16. Huang SF, Ko CW, Chang CS, Chen GH. Liver abscess formation after transarterial chemoembolization for malignant hepatic tumor. Hepatogastroenterology 2003; 50:1115-8.

17. de Baere T, Roche A, Amenabar JM, Lagrange C, Ducreux M, Rougier $\mathrm{P}$, et al. Liver abscess formation after local treatment of liver tumors. Hepatology 1996; 23:1436-40.

18. Ebisutani C, Sato S, Nishi K, Inoue H, Yoshie T, Kinoshita Y. Antibiotic prophylaxis in transcatheter treatment of hepatocellular carcinoma: An open randomized prospective study of oral versus intravenous administration. Intern Med 2010; 49: 1059-65.

19. Zheng PF, Zhang JS. The meta analysis of recent complications after the interventional therapy in the advanced hepatocellular carcinoma patients. Shanghai J Prevent Med 2007; 19:517-9.

20. Fiorentini G, Aliberti C, Tilli M, Mulazzani L, Graziano F, Paolo G. Intra-arterial infusion of irinotecan-loaded drug-elutin beads (DEBIRI) versus intravenous therapy (FOLFIRI) for hepatic metastases from colorectal cancer: Final results of a phase study. Anticancer Res 2012; 32:1387-95. 